Pedagogía y Saberes n. ${ }^{\circ} 55$

Universidad Pedagógica Nacional

Facultad de Educación. 2021. pp. 179-191

\title{
Perspectivas de la filosofía de la educación (2003)
}

Omar Camilo Moreno Caro*

\section{Para citar este artículo}

Wilson, J. (2021). Perspectivas de la filosofía de la educación (Trad. O. Moreno Caro). Pedagogía y Saberes, (55). https://doi.org/10.17227/pys.num55-12282

\section{Fuente}

Wilson, J. (2003). Perspectives on the Philosophy of Education. Oxford Review of Education, 29(2), 279-273.

Pregrado en filosofía de la Universidad Nacional de Colombia y maestro en filosofía de la Universidad Nacional Autónoma de México. Correo electrónico: ocmorenoc@unal.edu.co 


\section{A}

Cuando hablamos de la psicología o la sociología de la educación o de alguien que estudia la educación desde un punto de vista histórico o económico sabemos bastante bien de qué y de quién estamos hablando. Es claro que hay una serie de disciplinas, formas de pensamiento o tipos de investigación que podemos aplicar a la educación, cada una tiene sus propios procedimientos o principios heurísticos, su propia materia y sus propios criterios de éxito. En pocas palabras, cada una tiene su propia lógica interna. Además, estas disciplinas son relativamente homogéneas cuando se ponen a prueba en campo, por ejemplo, podemos encontrar a un historiador contemporáneo de la educación haciendo el mismo tipo de razonamientos que un historiador de la educación hace cien años; del mismo modo, podemos reconocer que Pitágoras y Poincare, o Newton y Einstein, se dedicaban a investigaciones similares, a las matemáticas y a la física respectivamente. Aunque sus respuestas fueran diferentes, ellos se hacían el mismo tipo de preguntas y usaban métodos similares para responderlas.

Sin embargo, podemos constatar que esto no aplica para lo que llamamos "filosofía de la educación". De hecho, diferentes personas pueden asignar significados variados a esta expresión porque tienen perspectivas muy diferentes de lo que la filosofía de la educación es o debería ser. Difieren en cuanto a sus procedimientos, temas y criterios de éxito. Es más, puede que ni siquiera tengamos claro a quién contar como filósofo de la educación: Froebel, Freire, Foucault, Marx, Montessori y Mao Zedong tuvieron mucho que decir sobre la educación, pero ¿a cuál de ellos llamaremos filósofo? Nuestra comprensión de otras disciplinas relacionadas con la educación nos permite al menos plantear preguntas sobre su uso o valor, por ejemplo, a docentes en formación o profesores en ejercicio, a investigadores de la educación o a responsables políticos; pero, en el caso de la filosofía de la educación difícilmente podremos hacerlo hasta que sepamos de qué estamos hablando.

Una razón importante de esta incertidumbre es la ausencia de una tradición intelectualmente respetable y bien establecida. Eso puede ser en parte un accidente histórico: Aristóteles estableció la agenda para muchas ramas de la filosofía, al menos en el mundo occidental, pero desafortunadamente lo que escribió sobre la educación se ha perdido en gran parte, por lo que es posible creer que la filosofía de la educación habría sido mucho más estable y estaría claramente definida si su trabajo se hubiera conservado. Ciertamente en este sentido la filosofía de la educación se encuentra mal posicionada con respecto a otras ramas de la filosofía, por ejemplo, frente a la filosofía moral y política, la metafísica, la epistemología y la lógica porque en ellas se pueden trazar fácilmente líneas de desarrollo desde Aristóteles hasta el presente. Sin embargo, desde mi punto de vista hay razones más profundas por las que no nos podemos apoyar en una tradición de la manera en que sí lo podemos hacer en el caso de la matemática, cuya tradición se remonta al menos hasta Euclides y Pitágoras.

Parte del problema es que la filosofía ha sido comúnmente definida y delimitada por una lista tradicional de preguntas de alto calibre: preguntas sobre la buena vida, el Estado ideal, la naturaleza de la realidad, la existencia de Dios, la presencia o ausencia de libre albedrío, la conciencia humana, la posibilidad de ciertos conocimientos y así sucesivamente. Las preguntas específicamente educativas (cualesquiera que sean) no están en esta lista, por lo que no es sorprendente que la mayoría de filósofos - tal vez todos- las hayan tomado como subsidiarias o dependientes de algunas de las preguntas filosóficas más grandes o centrales. En general, los filósofos se han preocupado por argumentar a favor de alguna doctrina de la condición humana o de algún régimen político o social ideal, y en todo caso han tratado la educación como una especie de apéndice (como lo hace Platón en República). Esto no es un buen síntoma para la filosofía de la educación entendida como una disciplina autónoma.

Quizás está relacionado con esto el hecho lamentable de que incluso los filósofos más agudos no están en su mejor momento cuando escriben sobre educación. Lo que Locke, o incluso Kant, dice sobre la educación está muy por debajo de sus altos estándares filosóficos y a veces sus contribuciones rozan lo absurdo. Los puntos de vista educativos de Bertrand Russell son difíciles de tomar en serio y lo mismo podría decirse de la propuesta de Platón de abolir la familia (Platón, 457b). En parte esto ocurre porque están presos de su propia ideología o incluso de sus propias fantasías sobre la condición humana, como es evidente en el caso de Rousseau; pero lo más grave es que escriben como si la educación no fuera realmente un tema filosófico respetable en absoluto, sino solo un campo de esparcimiento para la teoría utópica o los prejuicios personales. Son palabras fuertes y por supuesto hay honrosas excepciones, pero cualquiera que, quizás solo por un interés anticuario, se haya acercado a la literatura pertinente encontrará muy pocas cosas parecidas a tradición filosófica fiable en el sentido en que tenemos una tradición fiable en, por ejemplo, la filosofía moral desde Platón y Aristóteles, la cual es "estable" tanto en su objeto como en su método.

Así, no parece haber una sola perspectiva en la filosofía de la educación, ni siquiera un conjunto de perspectivas distinguibles. Más o menos todo vale, 
como puede verse en las actas más recientes de la Sociedad de Filosofía de la Educación de Gran Bretaña (2001). Las contribuciones son desconcertantes ("Influencias foucaultianas en el giro hacia la terapia narrativa", "La promesa de la Bildung", "Esperando en la Web", "Reconsideración de la visión de Rorty del irónico liberal como el ideal posmoderno de los educandos", etc.): no solo los temas varían, sino que no hay un único estilo o forma de discurso. Un forastero, o incluso un conocedor de las modas académicas, se puede sentir desconcertado tanto por la cuestión de qué se supone que es la filosofía de la educación como por la cuestión de cómo se supone que se debe tratar lo que se supone que es.

Por supuesto, es cierto que no hay una perspectiva pública bien establecida sobre la filosofía en general, por lo que en los estantes de las bibliotecas públicas las obras de filósofos respetables se encuentran junto a Pensamientos sobre la vida después de la muerte de Ada Astrologue. Pero, esto no es una excusa: un estudiante inteligente no tarda en identificar contribuciones serias a la filosofía de la ciencia, del derecho y de la moral porque las revistas especializadas dedicadas a estas ramas de la filosofía poseen una homogeneidad que está ausente en las revistas dedicadas a la filosofía de la educación.

Tampoco es una excusa el hecho de que, como se da ahora por sentado - véase la sección D de este escrito y a Partington (2001) - "el concepto de educación" pueda ser "discutible", lo que incluso privaría a la filosofía de la educación de cualquier tema fijo o identificable. Incluso si esa opinión fuera cierta (o inteligible), esto también podría aplicar a los conceptos de ciencia, de derecho o de moralidad y, sin embargo, en esos casos, esta opinión es completamente inofensiva. Tenemos que afrontar el hecho de que parece haber algo peculiarmente problemático o confuso en la filosofía de la educación, de modo que pareciera que no tenemos ningún terreno sólido bajo nuestros pies. Desde este punto de vista, no es sorprendente que la filosofía de la educación parezca no tener ninguna clase de impacto en la práctica educativa.

\section{B}

Espero haber demostrado hasta ahora que no podemos confiar en ninguna concepción tradicional o institucionalizada o - por así decirlo — prefabricada de filosofía de la educación. Así, tenemos que empezar más o menos desde cero, lo que nos obliga a preguntarnos qué ganamos al preferir una concepción sobre otra o al adoptar una perspectiva en lugar de otra. Si no tenemos criterios públicos preestablecidos o bien establecidos que nos guíen, ¿qué criterios debemos utilizar para dife- renciar y evaluar las diversas perspectivas? Tal vez no podamos responder plenamente a esto hasta que hayamos utilizado realmente algunos criterios y esbozado algunas perspectivas. Pero una respuesta corta podría ser esta: queremos ser capaces de identificar una actividad a) que puede ser llamada justamente "filosofía de la educación" (de lo contrario, nuestra descripción de la misma será engañosa); b) que no hemos identificado ya bajo algún otro título (de lo contrario no habremos ganado terreno ni arrojado nueva luz sobre nada); c) que es, en cierto modo, claramente diferente de otras actividades y suigeneris (de lo contrario no la habremos distinguido de otras actividades); y d) cabe esperar que sea una disciplina que tenga algún tipo de utilidad o valor para quienes se dedican a la empresa práctica de la educación, ya sea para los alumnos-profesores, para los profesores en ejercicio, para los responsables de la formulación de políticas educativas o para los investigadores en materia de educación.

Es sorprendente que los llamados "filósofos de la educación" digan poco o nada sobre su disciplina que satisfaga estos criterios. Tienen mucho que decir sobre filosofía de la educación, pero no nos dicen en qué consiste realmente. Así, en lo que podría suponerse que es un compendio razonablemente autoritario (en la serie Oxford Readings in Philosophy), Peters (1971) nos dice que en su introducción editorial que esta disciplina debería ser "específicamente filosófica", "sistemática", preocupada por "el análisis y el argumento" y no solo la vieja mezcla de exposición histórica y recomendaciones sobre políticas y prácticas". White (en Mills, 2001) dice que la filosofía de la educación "puede iluminar importantes cuestiones educativas"; Kohli (1995) que contribuye a la "salud y vitalidad de la teorización educativa"; Carr (1995) que debería ofrecer "la oportunidad de un pensamiento educativo radical"; el propio Mills (2001) que tiene "sus propias técnicas de probidad" de modo que "el filósofo de la educación es alguien que puede dominar el contenido y los métodos de indagación crítica [...] y proporcionar ideas iluminadoras" y así sucesivamente. Pero nada de esto nos dice qué tipo específico de "iluminación" proporciona el filósofo o simplemente cómo contribuye a la "teorización educativa", o precisamente cuáles son sus "técnicas de probidad" o "métodos de investigación crítica". Por supuesto, se trata de golpes bajos, sacados de contexto; pero incluso así es justo decir que los filósofos de la educación no nos han dado una explicación clara de cómo se supone que funciona realmente su disciplina: de los tipos de argumento que utiliza, la evidencia que consideran relevante, sus test de verdad y falsedad, sus criterios de éxito o fracaso, el estatus de sus propias proposiciones y pronunciamientos y su lógica interna en general. 
Esto es comprensible, si no excusable, pues cualquier relato de este tipo puede ser muy controversial y es natural que los filósofos quieran saltarse cualquier exposición de lo que se supone que hacen y seguir con lo que están haciendo. Nos queda inducir sus perspectivas (conscientes o inconscientes) sobre la filosofía desde su trabajo y a veces tales inducciones pueden ser bastante fáciles de hacer. Pero, una vez más esto significa que no tenemos una perspectiva bien establecida desde la que podamos trabajar: del mismo modo que no hay una tradición homogénea en lo que hacen, tampoco hay una tradición homogénea en las propuestas teóricas (extremadamente pobres) que surgen de su trabajo. Como era de esperar, los filósofos de la educación tienen en común su opinión de que la filosofía de la educación nos brinda algún tipo de luz, pero no nos dicen qué tipo de luz arroja ni cómo la arroja.

\section{C}

Me he esforzado por hacer énfasis en lo confundidos que estamos porque me parece escandaloso que nos quedemos satisfechos con el estado actual de la cuestión. Mi intención no es promover una regulación que prohíba la filosofía de la educación, de la misma manera que los oponentes políticos de Sócrates trataron de prohibir la práctica de la lógica; en todo caso, si no sabemos lo que se supone qué es la filosofía de la educación, dicha ley sería tan ininteligible como arbitraria. Pero, tampoco parece haber ninguna razón suficiente por la que debamos promoverla, institucionalizarla o pagar a la gente para que estudie esta disciplina. Tal vez esta no sea más respetable que la astrología y los filósofos de la educación no sean más que fanáticos, culebreros o vendedores de humo. Tal vez sea esta la opinión -no expresada frecuentemente debido a que se mantienen las buenas costumbres en la académica-, de los pobres educadores que una y otra vez sienten que no han obtenido en absoluto ningún beneficio de esta disciplina.

La única forma de avanzar en esta cuestión es tratar de identificar una serie de actividades diferentes, teniendo en cuenta los criterios a, b, c y d mencionados la sección B y ver en cuál de ellas podemos poner todas nuestras esperanzas. Intentaré esto más adelante, aunque la lista está lejos de ser exhaustiva y sus puntos no están tan elaborados como merecen. Todo lo que pretendo lograr aquí es que su descripción pueda servir de base para una taxonomía más seria y detallada y tal vez lograr dar algunas indicaciones generales de cómo serían algunas de las perspectivas que vale la pena considerar.

\section{La filosofía como un credo personal}

Hay un sentido bien reconocido de filosofía en el que hablamos de "su ('mi', 'tu', 'la de Platón', 'la de Dewey', etc.) filosofía de la educación", en contraposición a la filosofía de la educación. Este sentido de entrada le da la espalda a la filosofía de la educación como una disciplina consolidada y la asume como una posesión personal. No podemos hablar de "mi ('su') historia o economía de la educación" del mismo modo en que tampoco podemos hablar de "mi ('su') matemática" o "mi física nuclear". Obviamente, esto ocurre porque vemos la historia o la economía, como las matemáticas o la física, más como disciplinas que como posesiones personales: no son cosas que se posean, sino saberes que se ponen en práctica o disciplinas que se aprenden.

En este sentido, filosofía es aproximadamente equivalente a "ideales" o "ideología" o a cierto sentido de "teoría". Tengo un conjunto más o menos bien articulado de valores educativos y prácticas predilectas a las que refiero si se me preguntan cuál es "mi filosofía de la educación". Cualquiera puede las opiniones de algún conocido filósofo de la educación - como puede ser Platón, Dewey o Montessori- o mencionar alguna categoría general de opiniones descrita como "progresista", "inclusiva" o hablar del "ideal integral de la educación"; además, en este caso no importa si las opiniones son más o menos concretas o quizá solo son compartidas por algunos colegas que trabajan en la misma escuela. Es una cuestión abierta cuán bien articuladas o estructuradas tienen que estar tales opiniones para que cuenten como una "filosofía" de la educación, pero en todos los casos incluirán alguna mezcla de hechos, valores, métodos y prácticas en los que cada quien confía en el momento de entrar en un aula.

La mayoría de los educadores tienen una filosofía de la educación en este sentido y en cierto modo resulta deseable - incluso inevitable- que lo hagan. Es difícil pensar cómo podrían prescindir de algún conjunto coherente de principios y prácticas, ya sea que merezcan o no el título ligeramente magnífico de "ideología" o "teoría". La única alternativa que le queda a los educadores es hacer sus elecciones más o menos por azar, por impulso o simplemente por costumbre, aunque corran el riesgo de finalmente carecer de cualquier tipo de coherencia o integridad. También podemos elogiarlos por su coherencia e integridad, incluso cuando desaprobamos el contenido de su filosofía; por lo menos ellos representan algo, tienen algo por verdadero y quieren estar a la altura de su profesión. 
También podemos pensar que hay algún valor en la actividad (o en aquello que podríamos llamar razonablemente "disciplina") que les ayudó a elegir, modificar o articular su filosofía de la educación. Por medio de la construcción de varias teorías o filosofías de la educación, los filósofos profesionales podrían ofrecer a los educadores una gama de posibilidades que probablemente ellos mismos no hubieran sido capaces de concebir por sí mismos; algo así como cuando los supermercados ponen en sus estantes una amplia gama de productos que pueden atraer a los consumidores; o como cuando algunos padres bonachones invitan a personas del sexo opuesto a conocer a sus hijos e hijas para que aumenten la gama de sus experiencias y puedan incluso encontrar una persona para enamorarse o casarse; o quizá, un paralelo más cercano es cuando una amplia gama de diferentes religiones puede ayudar a las personas a encontrar la religión que más les convenga o les atraiga.

Aunque esta idea ("mercantil") de la filosofía de la educación no es para nada desdeñable, es cierto que tiene límites obvios. Pues, tan pronto como se pregunta si sería correcto para adoptar una filosofía particular, es necesario algo más más que su mera presentación; del mismo modo que se necesita más que conocer personas del sexo opuesto para decidir casarse o no con uno de ellos. Es posible enamorarse involuntariamente, también puede haber una atracción involuntaria a alguna filosofía o a alguna religión, pero esta elección debería hacerse de forma mucho más cauta, nadie quiere ser esclavo de sus instintos o sentimientos. Es necesaria algún tipo de crítica objetiva, es decir, la aplicación de alguna disciplina que permita determinar si los componentes de una determinada filosofía de la educación son verdaderos o falsos, correctos o erróneos, sensatos o equivocados. Que sea atractiva o no, no es la única consideración, de hecho, tal vez no sea una consideración racional en absoluto, ya que algo puede atraer sin tener razón o tener razón sin ser atractivo todavía.

Pero tan pronto como tomamos en serio esta última idea, nos movemos hacia un sentido diferente de filosofía. Ahora, "filosofía" será el nombre de una disciplina, no de un credo personal. Se trata de algo que está por encima de las filosofías particulares y que puede ayudar a evaluarlas, una actividad por derecho propio. La "filosofía de la educación" (en contraposición a mi filosofía de la educación) dará derecho a esta actividad, como la "historia" o la "economía" de la educación dan derecho a diferentes actividades. Ahora queremos saber en qué consiste esta actividad y cómo llevarla a cabo con éxito. Por lo tanto, en este punto ya hemos adoptado una perspectiva diferente a partir de un sentido diferente de "filosofía de la educación".

\section{La filosofía como una disciplina sobre juicios de valor}

Quizás lo primero que valga la pena saber es si los juicios de valor de una determinada filosofía educativa son correctos. ¿Se está en lo cierto cuando se piensa que a los niños se les debe dar más libertad de expresión o una disciplina más severa, que es más importante que aprendan esta materia en vez de aquella, que el "ethos" de sus escuelas debe ser de uno u otro tipo, que deben ser seleccionados sobre este o aquel principio o (si eso fuera posible) no ser seleccionados en absoluto? Todos tenemos juicios de valor sobre estos asuntos, pero ¿puede la filosofía ayudar a evaluarlos?

Como ya hemos visto en la sección anterior, la filosofía no puede hacer esto meramente presentando alternativas: esto no ofrece ninguna razón para elegir entre ellas. Podría aportar proporcionando hechos empíricos, por ejemplo, datos sobre las consecuencias reales de dar a los niños más o menos libertad o de enseñarles una asignatura en lugar de otra. Sin embargo, esto no parece particularmente "filosófico" y me ocuparé de ello en la sección 3 más abajo. Aparte de esto, todo lo que la filosofía puede hacer (si es que puede hacer algo) es explicar los criterios racionales que son aplicables a los juicios de valor para saber cómo evaluarlos. No lo hará directamente diciendo cuáles juicios de valor son razonables y cuáles no; esto, una vez más, no hace más que presentar un conjunto de "respuestas" a las que el filósofo da su sello personal de aprobación ¿Por qué se deberían asumir estas respuestas si estas no obedecen a un criterio racional? Todo el peso de la filosofía residirá entonces en dilucidar y justificar estos criterios: mostrará, por así decirlo, cómo manejar las creencias y los juicios de valor propios, de la misma manera que los principios de la ciencia muestran cómo manejar las creencias y los juicios sobre el mundo físico.

Podemos decir que esta disciplina se ocuparía de "la lógica de los juicios de valor" y esto, por supuesto, ha sido una preocupación filosófica perenne, incluso cuando ha dado lugar, o no, a algún criterio claro y generalmente acordado como el que necesitamos (parece bastante claro que no lo ha hecho). Obviamente, tal investigación es inmensamente valiosa porque sin ella no tenemos ninguna esperanza de saber si nuestros juicios de valor son acertados o erróneos. Por lo tanto, esto nos da una perspectiva justificable de la filosofía de la educación: la buscamos para que nos ayude con nuestros juicios de valor, no entregándonos 
un conjunto de valores en un plato que simplemente tenemos que tragar, sino mostrándonos cómo operar razonablemente en este campo en particular.

Sin embargo, eso no es suficiente porque, además de los valores educativos, hay muchos tipos de valores diferentes, a saber, valores morales, políticos, estéticos, quizás religiosos, entre otros. De hecho, no está nada claro qué tipo o género de valores son especialmente relevantes para la educación y hasta qué punto se conectan con los demás. Constantemente, los filósofos han relacionado estrechamente los valores de la educación con los valores morales o éticos (como en el título de Peters para su obra maestra, Ética y Educación (1965)), pero no debemos dar esto por sentado. ¿Existen valores educativos por derecho propio, tal vez fundamentados en el concepto mismo de educación, o los valores educativos solo son una mezcla de valores morales, políticos y demás que surgen en las prácticas educativas? Dejo esta pregunta a un lado - ya la he discutido en Wilson, (1979) - , mi punto con esto es que es necesario responder a la pregunta de si la filosofía de la educación debe ser una disciplina sui generis. Porque, si no hay nada peculiar en los juicios de valor propios del campo de la educación, entonces no habrá (al menos en este aspecto) nada peculiar en la filosofía de la educación: se reducirá a la filosofía de los juicios de valor en general. Queda mucho trabajo por hacer en este respecto como para afirmar que la filosofía de la educación "se ocupa de los valores", "desafía los supuestos sociales" o "critica nuestros presupuestos educativos básicos". La filosofía moral, la filosofía política y la estética son ramas de la filosofía distinguibles y reputadas en parte porque tenemos alguna idea (aunque no muy clara) de cómo distinguir los valores morales, políticos y estéticos. Si pudiéramos hacer lo mismo con los valores educativos, sería un gran paso adelante. En la actualidad, incluso la frase "valores educativos" parece bastante misteriosa o, por así decirlo, vacía. Sabemos que la bondad y el coraje tienen un valor moral; la libertad y la justicia un valor político; y la elegancia y la belleza un valor estético, pero ¿podríamos hacer una lista de "valores educativos" de la misma manera? Sin embargo, si se cree en la primera frase de la Ética a Nicómaco de Aristóteles (“Todo arte y toda investigación, así como toda búsqueda o comprensión práctica, parece tener como objetivo algún bien"), la educación debe tener presumiblemente bienes y valores propios.

\section{La filosofía como disciplina empírica}

Si la filosofía puede ser de utilidad, es de esperar que genere algún tipo de verdad, es decir, debería poder decirnos que las cosas son así y no de otro modo.
Pero, inmediatamente nos encontramos con lo que seguramente debe ser el problema central que afecta a nuestra perspectiva de la filosofía: el problema de determinar qué tipo de verdad se supone que genera esta disciplina. El problema se agudiza y se hace evidente cuando consultamos la entrada del diccionario (Fowler et al., 1995) para la expresión filosofía:

el uso de la razón y la argumentación en la búsqueda de la verdad y el conocimiento de la realidad, especialmente de las causas, la naturaleza de las cosas y de los principios que rigen la existencia [...], el universo material, la percepción de los fenómenos físicos y el comportamiento humano.

Pero, ¿no es precisamente esto lo que hacen los científicos naturales?, ¿no es cierto que incluso allí donde el científico natural no tiene lugar, como en el caso del "comportamiento humano", existe una serie de profesionales competentes como científicos y psicólogos sociales, historiadores, sociólogos, antropólogos, ente otros?, ¿qué es lo que hace el filósofo que no esté tratado ya por alguna otra disciplina?

Todas las disciplinas mencionadas se ocupan de hechos empíricos, y por empírico entendemos aquí algo así como "contingente", es decir, hechos que podrían ser o podrían haber sido diferentes de lo que son o de lo que fueron, en el mismo sentido en que los hechos sobre los átomos que constituyen un compuesto podrían ser diferentes o los hechos sobre la Segunda Guerra Mundial podrían haber sido distintos. De este modo, necesitamos averiguar por experiencia o investigación empírica (del griego empeiria, "experiencia") lo que realmente son o fueron, del mismo modo que hacen los físicos e historiadores. Esto quizás podría contrastar con los hechos y verdades no empíricos, lógicos o "necesarios", por ejemplo, es un hecho o verdad que, si A es más grande que B y B más grande que $C$, entonces, $A$ es más grande que $C$. No obstante, no llegamos a este hecho o verdad por experiencia, parece ser un asunto de pura lógica y no podría ser de otra manera. Ahora bien ¿debemos concebir la filosofía como una disciplina empírica en este sentido?

A fin de cuentas, no importa qué tipo de hechos empíricos sean cuestionados. En la actualidad, los filósofos no investigan los constituyentes de los átomos o los acontecimientos ocurridos en la Segunda Guerra Mundial, dejan esto a los físicos o a los historiadores. Pero, se podría suponer que investigan las ideas, ideales, conceptos, concepciones o estilos de pensamiento adoptados o sostenidos por individuos y sociedades pasados o presentes, por ejemplo, las ideas detrás del movimiento romántico en el arte y la literatura, el antiguo concepto ateniense de democracia en comparación con el nuestro o la forma 
en que los victorianos en Inglaterra o los antiguos espartanos concebían la educación. Supongamos que tengo alguna descripción general o teoría explicativa sobre la sociedad o sobre el clima intelectual actual, por ejemplo, "la ética protestante", "posmodernidad", el "relativismo", el "consumismo" o lo que sea. Aquí trato con los hechos, aunque lo hago de una forma un tanto peculiar, pues los hechos son históricos, están limitados en el tiempo y por la cultura, es decir, podrían ser o haber sido de otra forma. Sumergiéndome en la literatura pertinente y en otros métodos, obtengo experiencia de una gran cantidad de datos y logro una nueva descripción o explicación que ofrezco como verdadera. Este tipo de investigación no es en principio diferente de lo que podrían hacer los historiadores de las ideas, los sociólogos u otro tipo de científicos sociales o teóricos. De hecho, esto es lo que realmente hacen, de modo que la "historia de las ideas" se reconoce como una rama respetable de la erudición empírica. Me preocupan los conceptos y las concepciones e ideas tal como aparecen en el terreno, en la mente de las personas o en la práctica social; si cometo errores, serán errores fácticos o empíricos.

No está en discusión que dichos hechos empíricos y teorías puedan ser útiles o esclarecedoras para un educador, pero esta perspectiva no deja lugar para la filosofía de la educación como una disciplina sui generis. De hecho, desde este punto de vista la filosofía se interpreta como una especie de sociología o historia peculiar o de alto nivel; esto no tiene nada de malo, pero en última instancia es una disciplina que no se distingue de lo que los sociólogos e historiadores ya hacen y han hecho. Puede que no haya verdades específicamente filosóficas en los escritos de, por ejemplo, Marx, Weber, Durkheim o Isaiah Berlin aparte de sus descripciones y explicaciones empíricas. Estas serán verificadas o falseadas en la forma en que las verdades empíricas son verificadas o falseadas, es decir, por la experiencia de los fenómenos tal como aparecen fundamentados en el mundo que experimentamos.

En parte, el deseo de escapar de esta perspectiva fue lo que motivó en los años 30 a algunos filósofos a considerar la filosofía bajo una luz diferente. Llegaron a verlo como un "análisis conceptual" y, sea lo que sea que hayan querido decir con esto, la filosofía no pretendía ser una investigación empírica de las concepciones particulares de los individuos o de las sociedades tal como aparecen desde el punto de vista que acabamos de describir (una descripción de varios conceptos de la democracia a través del espacio y el tiempo, por ejemplo). Utilizaron, o inventaron, un sentido diferente de "concepto", uno más ligado al significado público de las palabras en uso. De este modo, podían hablar del "concepto de X" en lugar de solo de "mi ('tu', 'su', 'el antiguo') concepto de X". Así pues, los conceptos no se interpretaron como fenómenos empíricos brutos que existen en la mente de las personas -el tipo de cosas que los psicólogos podrían investigar-, sino que se consideraron como conceptos representativos o coextensivos de rangos de significado público determinados por las palabras usadas.

Esta idea y todo el asunto del "análisis conceptual" o "filosofía lingüística", que tuvo su apogeo más o menos entre los años 1940-1970, ha sido ampliamente criticada por muchos motivos - véase, por ejemplo, Carr (1995). Pero, en este contexto, su dificultad más grave es que, al menos a primera vista, no ofrece realmente una escapatoria de lo empírico. Supongamos que "analizamos", explicamos o elucidamos el concepto "educación". Muestra de ello es que, como no se trata de ningún tipo de investigación histórica o sociológica, esta búsqueda equivale a una investigación sobre cómo se utiliza la palabra education ${ }^{1}$ en el inglés contemporáneo. Pero, esto no es un procedimiento menos "empírico" que los anteriores, de hecho, podría ser de otra manera porque es un hecho que las palabras tienen diferentes significados en ciertos idiomas naturales en momentos distintos. Esto es lo que los etimólogos y los creadores de diccionarios pretenden decirnos. Puede ser importante que entendamos plenamente lo que significan y, por ello, poder entendernos cuando las usamos, pero cualquier verdad sobre sus reglas de uso serán verdades empíricas, verdades sobre la forma en que funcionan los lenguajes naturales. Formarán parte de la lingüística, que es una disciplina empírica reconocida (o, al menos, la parte que llamamos "semántica").

En este sentido, esta perspectiva de la filosofía todavía la mantiene empírica y, por ende, no le da ningún estatus sui generis, tampoco logra distinguirla de los tipos de investigación ya clasificados ("historia", "sociología" o "lingüística"). Es claro que se requiere un movimiento más radical que intentaré describir ahora.

\footnotetext{
1 Nota del traductor. En este punto y de ahora en adelante he decidido mantener la forma original de aquellas palabras del inglés a las que el autor haga referencia directa o metalingüística. Esta decisión se justifica en la forma en que desarrolla su argumentación, pues de aquí en adelante va a jugar un papel central el uso de las palabras en un determinado idioma natural. Mi propuesta es que, en la medida en que evitemos traducir las palabras que utiliza y evitemos las referencias al español, será posible ver los verdaderos alcances y límites de la propuesta del autor.
} 


\section{La filosofía como una disciplina lógica}

La definición de filosofía en el diccionario como "el uso de la razón y la argumentación en la búsqueda de la verdad" nos dice algo importante: que la filosofía procede por razonamientos y argumentación en lugar de basarse en, por ejemplo, la intuición, la revelación divina o la aceptación acrítica de la autoridad. Lo que esta definición no nos dice es el tipo de razón, argumento o verdad que le interesa a la filosofía. El punto crucial es que no toda razón, argumento o verdad es empírica. Como ya hemos visto, no es una verdad empírica que, si A es más grande que B y B más grande que $C$, entonces $A$ sea más grande que $C$ y, además, no llegamos a esto por medio de razones o argumentos empíricos. Es más natural decir que alcanzamos esta conclusión por lógica. Lo mismo podría decirse naturalmente de otras verdades, incluyendo quizás las verdades de las matemáticas. Si tengo siete invitados y cada uno de ellos necesita tres copas de vino, sé que necesito veintiún copas de vino, es un hecho, pero no llego a él por la experiencia, sino por la lógica. Puedo aplicar la lógica al mundo empírico, a los verdaderos A, B y C, a los verdaderos invitados y a las copas; por eso la lógica y las matemáticas tienen un valor práctico. Sin embargo, sus verdades no dependen del mundo empírico.

¿De qué dependen? Es tentador decir que dependen del significado de ciertos términos, es decir, que debido a que "más grande que”, "7", “3” y "21" significan lo que significan, las verdades necesariamente se siguen. En un sentido esto es correcto: si "más grande que" significara algo diferente o si usáramos una notación matemática diferente (un sistema binario en vez de uno decimal, por ejemplo), ya no podríamos decir verdaderamente que $A$ es más grande que $\mathrm{C} o$ que $7 \times 3=21$. Esto se debe a que las verdades, para ser tales, tienen que ser declaradas en algún lenguaje o notación, de modo que los elementos del lenguaje o notación en los que las declaramos importan. Pero, en otro sentido no es correcto, obviamente las verdades no son una función de los hechos empíricos sobre lo que significa bigger than en inglés o de una notación matemática en particular. Podemos enunciarlas en otras notaciones y lenguajes como "A es más grande que B y B es más grande que C", etc. De la misma manera, se puede decir que las verdades de la lógica formal "dependen" del sentido de palabras como some, all, if, not, entre otras, pero eso no significa que dependan de su uso en el inglés.

Para tomar un ejemplo que tiene que ver con la filosofía de la educación, supongamos que empiezo con las palabras inglesas education, indoctrination, training y así sucesivamente y muestro que el significado y el uso de estas palabras en el inglés contemporáneo difiere, por ejemplo, que education cubre más terreno que training o lo que sea. Entonces, alguien puede decir "eso es solo un hecho empírico sobre el uso del inglés: ¿por qué debería tener alguna importancia filosófica?” Este individuo no habría captado el punto porque lo que he hecho no es solo para describir el uso del inglés, sino también para ilustrar una distinción que existe por derecho propio. Education es una cosa diferente de training y - si la conversación se lleva a cabo entre los angloparlantes- utilizo nuestro conocimiento mutuo del inglés para marcar esta diferencia. La conversación podría igualmente llevarse a cabo en francés o en cualquier otro idioma; tampoco importaría incluso si el idioma no tuviera paralelos lingüísticos o equivalentes a education y training - podría, si fuera necesario, inventar nuevas palabras para marcar la distinción. Se nos dice que algunas sociedades primitivas no hacían distinciones entre las exigencias de la moral, el derecho, la religión y las convenciones, sino que se basaban más bien en una noción general de lo que estaba bien o mal, hecho o no hecho, no obstante, esto no significa que esas distinciones sean irreales o inexistentes cuando una sociedad no las utiliza o no las incluye en su vocabulario.

En este sentido, la filosofía se ocupa del descubrimiento (y quizás también de la creación) de distinciones lógicas transhistóricas y transculturales, por ello, su labor no se desarrolla en el terreno denotado por el sentido normal de "empírico". Los filósofos inspeccionarán las palabras en lenguajes naturales solo como una especie táctica para llegar a las distinciones. Para ilustrar esto, podemos pasar a otro ejemplo que empieza desde el otro extremo del proceso. Existe claramente una diferencia que puede describirse en inglés o en cualquier otro idioma entre: a) una clase de niños que se mantienen bajo control y sin problemas por algunos (cualesquiera) métodos - pueden ser aterrorizados o encantados, fijados con pegamento a sus asientos o sedados por tranquilizantes-,y b) una clase de niños que aceptan y actúan según el principio de obedecer a una autoridad legitimada (el maestro) solo porque la autoridad es legítima. Ahora bien, una forma de dilucidar esta diferencia es preguntar qué significa well-disciplined en inglés: podríamos, por ejemplo, sugerir que el término no se utiliza correctamente de (a) sino solo de (b). De la misma manera, podríamos distinguir entre a) una norma social, un mandamiento judicial o una ley que establece que los alumnos no deben intimidar a los demás ni consumir drogas y b) una esperanza o deseo piadoso, un ideal o la preferencia por no intimidar a los demás ni consumir droga; en este caso podemos sugerir que a) implica algún tipo de castigo, pena o sanciones que se aplican 
característicamente al comportamiento prohibido, mientras que en el caso de b) no es necesario. Se podría llegar a esa distinción examinando las diferencias de significado de términos como rule, law, hope, ideal y así sucesivamente. Pero, las diferencias en sí mismas no dependen de su significado; si alguien dijera que no utiliza (o no quiere utilizar) los términos en su sentido público habitual, aún podría ser llevado a reconocer las diferencias incluso con otros nombres.

Ahora podemos entender por qué los filósofos se vieron tentados a hablar del "concepto de $X$ " en lugar de solo del "significado del término $X$ ", es claro que no querían poner todas sus esperanzas en hechos empíricos sobre los significados de las palabras. También podemos observar de dónde surge la tentación de darle un peso considerable al "lenguaje ordinario"; creían (seguramente con razón) que algunas o muchas distinciones probablemente ya estaban marcadas $y$, por lo tanto, eran accesibles en lenguajes naturales que se utilizaban con éxito para comunicarse en la vida cotidiana. Hasta dónde deberían ceder a una u otra tentación es una cuestión abierta. Yo mismo pienso que, para sus propósitos, hablar de "conceptos" es peligrosamente engañoso (porque, como hemos visto, el "concepto" también tiene un sentido diferente y empírico, aproximadamente equivalente a "idea" o "concepción" con el que se puede confundir el sentido del filósofo). Por otra parte, la atención prestada al "lenguaje ordinario" parece razonable y no tiene por qué implicar la suposición de que el lenguaje ordinario es autoritario o correcto. (Si, como dice Carr (1995, p. 22), "el análisis conceptual se negó resueltamente a ver el sentido de cuestionar la adecuación de los conceptos establecidos con la realidad", entonces no deberían hacerlo y dudo que algún respetable "analista conceptual" lo haya hecho realmente). La cuestión no es solo que el lenguaje ordinario sea un depósito natural de distinciones importantes, también es que las cuestiones filosóficas surgen en un lenguaje natural y tienen que ser discutidas en un determinado lenguaje natural. Así pues, si mi esposa quiere saber si lo que siento por ella es realmente amor (en contraposición a admiración, afecto, amistad o lujuria), es difícil ver cómo se puede responder a esa pregunta sin examinar los significados de esas palabras junto con examinar mis sentimientos y comportamientos reales. Aunque tengamos que importar términos de un idioma diferente (por ejemplo, el griego eros, philia o agape, que señalan diferentes tipos de amor), la conversación será en un idioma determinado e implicará la inspección de las palabras. No obstante, la discusión no se tratará de palabras inglesas (o griegas), se tratará de diferentes tipos de sentimientos para los que las palabras son solo marcadores.
Tal vez los "analistas de conceptos" o los "filósofos lingüísticos" no fueron del todo claros en este punto o no lo expresaron claramente, esa puede ser una de las razones por las que ese tipo de filosofía está pasada de moda. Tampoco propongo su resurgimiento. Sin embargo, sí parece que la perspectiva de la filosofía como disciplina lógica, en el sentido en que la he descrito, da a la filosofía un estatus sui generis, principalmente porque la distingue de las disciplinas empíricas por una parte y de la ideología personal por otra. Por supuesto, incluirá la lógica de los juicios de valor (sección 2), pero no solo eso; finalmente, su objeto natural serán esas distinciones lógicas (por no decir conceptuales) que parecen particularmente pertinentes para la educación. Evidentemente hay muchas de ellas (ya he dado uno o dos ejemplos), por lo que la filosofía de la educación no se quedará corta de material: más bien hay una desbordante riqueza, pues casi todas las cuestiones educativas importantes giran en torno a la aclaración de distinciones y alrededor del conocimiento de los hechos empíricos.

Más que esto: si ahora nos preguntamos hasta qué punto la filosofía de la educación entendida así es útil o valiosa, la respuesta no se debe buscar lejos. En términos generales, la respuesta es que las distinciones y categorías que utilizamos dirigen en gran medida nuestro pensamiento, y nuestro pensamiento orienta en gran medida nuestra práctica, a saber, nuestra organización, las líneas de nuestra investigación y lo que realmente hacemos en el aula. La forma en que un profesor concibe la "disciplina" afectará profundamente lo que pretende lograr en el aula y lo que el investigador educativo considera que es el tema de su investigación. Por ejemplo, lo que decidamos entender por espiritualidad, ciudadanía o educación moral regirá lo que realmente hagamos bajo esos títulos. La forma en que clasificamos a los alumnos a partir de rótulos como necesidades especiales, discapacitados o con dificultades de aprendizaje en sí mismo determinara cierto tipo de alumnos para cierto tipo de trato. No necesito profundizar más en este punto.

Finalmente, a partir de lo que he dicho, será evidente que esto no representa ningún intento de monopolizar la palabra filosofía. La palabra tiene varios usos y ninguno de ellos es "mejor" o "peor" que cualquier otro. No se trata de eso, más bien el asunto es saber si podemos caracterizar una disciplina que pueda distinguirse de las demás y que sea manifiestamente pertinente para la práctica de la educación, para la cual la palabra filosofía sea un marcador plausible. Eso es lo que he tratado de hacer en la sección 4 y ese es el tipo de perspectiva sobre la filosofía de la educación que defiendo como la más fructífera. No estoy en desacuerdo con otras actividades que puedan llevarse 
a cabo bajo ese marcador, pero, si esta actividad en particular no fuera entendida y promovida, creo que sería una gran pérdida tanto para la teoría como para la práctica de la educación.

\section{D}

Todavía tenemos que considerar la cuestión de cuál es objeto que se supone que debe tratar la filosofía de la educación. Se trata de una cuestión diferente a las anteriores porque en los apartados 1 a 4 nos hemos concentrado en el método y no en el objeto o tema de estudio. En la útil terminología de Hirst (1970), no hemos considerado la filosofía como un campo de pensamiento sino como una forma de pensamiento. La distinción es crucial y no dejaré de insistir en ella. Los campos de pensamiento son, por así decirlo, materia bruta; por otra parte, las formas de pensamiento son las disciplinas lógicamente distinguibles que aplicamos a los campos. Así, nuestro objeto de estudio o tema puede ser "sexo" o "catedrales", pero podemos traer considerar el sexo desde la psicología, la biología o la filosofía moral; además, podemos estar interesados en las catedrales desde los puntos de vista del ingeniero de la construcción, del religioso o del economista cuya preocupación es hacerlas financieramente viables. De la misma manera, la historia, la psicología, la sociología y la filosofía de la educación tienen que ver con la educación y, por ende, la tienen como su objeto de diferentes maneras.

Esto es importante para entender la noción de "perspectiva" de la filosofía de la educación. Algunas personas pueden querer interpretar la filosofía en términos muy diferentes a los descritos en las secciones 1 a 4, a saber, no la consideran en cuanto a sus métodos o procedimientos, sino con relación a su objeto. Así, estas personas podrían considerar que el trabajo de la filosofía es dar respuesta a ciertas preguntas generales, abstractas o importantes: preguntas sobre el sentido de la vida, la existencia de Dios, el libre albedrío y la conciencia humana, la "realidad", los "valores últimos", etc. Además, podrían señalar algún tipo de tradición en apoyo de este punto de vista porque los filósofos han estado característicamente preocupados por este tipo de preguntas y han heredado una agenda a partir de estas preocupaciones. Pero, incluso si pudiéramos estar de acuerdo en los asuntos que debían figurar en tal agenda, la pregunta seguiría siendo cómo se supone que los filósofos deben responderlas: por medio de qué métodos, qué tipos de evidencia, qué tipos de razonamiento y así sucesivamente. Entonces, nos vemos obligados a considerar qué métodos, tipos de pruebas y tipos de razonamiento - es decir, qué formas de pensamiento- hacen justicia a la investigación.
La filosofía de la educación no encaja bien en la agenda filosófica tradicional porque las preguntas que incluye esta agenda casi nunca son específicamente educativas. Sin duda, algunas cuestiones educativas están en algún momento conectadas o relacionadas con cuestiones muy generales sobre el significado de la vida, la naturaleza del conocimiento, la condición humana, los "valores últimos", o incluso quizás la existencia de Dios, sin embargo, eso difícilmente da a la filosofía de la educación una materia específica o sui generis. Si preguntamos qué trata la filosofía de la educación, es más sencillo decir (aunque sea aburrido) que se trata de educación de la misma manera que decimos que la filosofía de la ciencia trata de la ciencia o la filosofía moral de la moral. Entonces tenemos la tarea (menos aburrida) de decir exactamente lo que vamos a incluir y a excluir bajo el título de "educación".

Aquí debemos comenzar reconociendo que no todo cuenta como educación. Si alguien dijera que se dedica a la educación cuando en realidad está masacrando judíos o comiendo pescado y patatas fritas, deberíamos pensar que su diccionario (si no su cabeza) necesita un examen. Lo mismo ocurre a la inversa: sería un sinsentido que un profesor o un alumno que imparte o toma algún curso organizado para aprender dijera que no se dedica a la educación. Reconocemos los casos centrales o paradigmáticos que deben incluirse sin dudar y otros casos que deben excluirse sin pensarlo. Sin duda habrá casos límite sobre los que no podremos estar seguros (¿los conserjes de las escuelas están "comprometidos con la educación"?, ¿qué hay de los arquitectos de las escuelas o los proveedores de comidas escolares?). Alguna vez ocurrió que una persona que fabricaba overoles para los ministros bautistas que practicaban la inmersión completa en el agua afirmó estar trabajando "en el campo de la religión", pero no debemos considerar esa conexión como suficiente para justificar su afirmación. Muchas cosas están relacionadas con la educación, sin embargo no son parte de ella.

Parte de la tarea de la filosofía de la educación consiste identificar claramente su propio objeto y el paso más natural que hay que dar, al menos inicialmente, es examinar lo que significa "education" en el inglés contemporáneo con la esperanza de identificar los parámetros lógicos de una actividad o empresa distinguible de otras actividades o empresas, independientemente de que exista o no en otros idiomas una palabra para hacer referencia a esta (l'education, Bildung, paideia, etc.). En este punto, deberíamos seguir los procedimientos filosóficos descritos en la sección 4, buscando establecer distinciones transhistóricas y transculturales y, a partir de esto, dar una descripción clara de aquello para lo que education 
es, en el inglés contemporáneo, un marcador plausible (al igual que hemos hecho con philosophy). Así, podríamos llegar a alguna definición o postulado como "un proceso de aprendizaje serio y sostenido en beneficio de las personas, que los lleva por encima de aquello que naturalmente podrían haber hecho por sí mismos". No voy a argumentar aquí a favor de esta explicación, ya que lo he hecho en otros lugares (Wilson, 1979), no obstante, al menos deberíamos estar de acuerdo en que la educación está centrada en el aprendizaje de la misma manera que la medicina está centrada en la salud o la economía en la forma en que se produce el capital.

¿Qué vamos a hacer ahora con la idea de que "el concepto de educación es discutible"? Los creadores de esta idea (Gallie, 1968) tuvieron razones para llamar la atención sobre el hecho de que diversas personas tienen y han tenido diferentes conceptos, ideas, ideales o imágenes de cómo debería ser la educación, del mismo modo que tienen diferentes concepciones del cristianismo, de la democracia o de un matrimonio feliz. "Concepto" se utiliza aquí de forma empírica, como hemos explicado anteriormente, porque señala a una imagen que condensa un conjunto de valores y prácticas que existe en la mente de un individuo determinado y a la que él atribuye el término educación. Por supuesto, tampoco es cierto que cualquier concepto de cualquier otra cosa cuente como un concepto válido de educación, por lo que es claro que el significado público de la palabra pone algunas restricciones al rango de las concepciones válidas. Por ejemplo, si alguien dijera que su concepto de la educación implica la masacre de todos los primogénitos, como Herodes, no podríamos entenderlo. Sin embargo, es claro que dentro de ese rango que deja el uso público del término hay mucho espacio para diferentes ideas acerca de qué tipo de aprendizaje debe promoverse, cómo debe ser impartido el programa de estudios o por qué criterio los niños deben ser agrupados en las escuelas, y así sucesivamente. Estas ideas pueden ser vistas como opuestas entre sí y, por lo tanto, son "discutibles" del mismo modo que las diferentes ideas acerca del cristianismo, la democracia o de un matrimonio feliz pueden ser rivales.

Sin embargo, no debemos confundir este sentido empírico con el otro sentido de "concepto" que se encuentra ligado a los significados públicos de las palabras. La diferencia es evidente en el caso de "un matrimonio feliz" o de "un buen marido". Cada uno puede tener diferentes concepciones de lo que es un matrimonio feliz o un buen marido, pero compartimos un entendimiento común de lo que significan realmente las frases "un matrimonio feliz" o "un buen marido". Incluso podemos ponerlas con confianza en otros idiomas o explicar su significado a los niños pequeños. De este modo, también podemos explicar lo que significa "educación" sin recurrir a nuestros valores particulares o a nuestras prácticas preferidas que nos gustaría ver relacionadas con esta palabra. En pocas palabras, podemos discutir sobre (a) lo que significa bueno, feliz o educación o (b) sobre qué cosas o personas son buenas, promueven la felicidad o deberían incluirse bajo el rótulo de educación; se trata de dos puntos de vista diferentes. Por supuesto, puede haber opiniones rivales sobre (a), por ejemplo, yo podría decir bueno, feliz o educación de una manera y el lector de otra; pero las ventajas y desventajas de estos puntos de vista se determinarían mirando los significados de las palabras y no nuestros ideales personales o creencias empíricas.

Como no podemos hacer que las palabras signifiquen lo que nos gusta, el objeto de la filosofía de la educación será aquello que signifique la palabra educación. Esto incluye una investigación de lo que realmente significa y, quizás, esto también implica una investigación de la manera en que la gente ha concebido la educación (aunque eso, como hemos visto antes, será una especie de investigación empírica, como una historia o sociología de las ideas). Todavía es posible que los filósofos de la educación se desvíen de este camino, de hecho, lo hacen a menudo en la práctica. Por un lado, se ocupan de asuntos que van mucho más allá de su responsabilidad, interpretando así su tarea de forma demasiado amplia, por otro lado, evitan los asuntos que efectivamente son su responsabilidad, interpretando su tarea de forma demasiado estrecha.

Ilustraré este punto por medio de algunos ejemplos concretos, si: 1) estoy investigando si un determinado sistema de escolarización hace a los niños más felices o más sanos, si les da más oportunidades de movilidad social o de éxito en la vida posterior o si es más justo o equitativo o democrático, ¿qué razón hay para decir que en estas investigaciones me preocupa la filosofía de la educación? En este caso, no estoy preocupado por la escuela como institución de aprendizaje, sino como institución social o política. Es una cuestión educativa si un sistema de escolarización promueve el aprendizaje de los alumnos de manera efectiva, por otra parte, si promueve la democracia o la igualdad es una cuestión política. Mucho de lo que se hace pasar por filosofía de la educación es realmente un asunto político, social o de algún otro tipo de filosofía (y no es para menos). No obstante, no tiene derecho a ese título si no está directamente relacionado con el aprendizaje, pues de eso se trata la educación. Pasa exactamente lo mismo 
si tengo interés en la arquitectura o en el diseño de las escuelas. Como filósofo de la educación lo que podría preocuparme es si uno u tipo de construcción o diseño promueve el aprendizaje de los alumnos (y no su costo o méritos estéticos).

Por otra parte, 2) si interpreto la educación simplemente en términos de algunas de sus manifestaciones o prácticas culturales - un sistema particular de escolarización, determinados exámenes públicos o las normas y reglamentos establecidos por gobiernos particulares en momentos particulares-, estaré ciego a muchas cuestiones que como filósofo de la educación debo afrontar. Del mismo modo, si interpreto la religión simplemente en términos de las religiones que se practican en mi propia sociedad o en cualquier sociedad particular, estaré ciego a cuestiones más generales y transculturales propias de la filosofía de la religión. La educación no se puede traducir en una serie de prácticas culturales concretas como tampoco la ciencia es equivalente a lo que hacen determinados científicos en determinados momentos y lugares. Estos términos denominan lo que podríamos llamar empresas trascendentales que se realizan o practican (más o menos adecuadamente) en situaciones concretas, pero que no se reducen a una determinada puesta práctica. Es una cuestión sin resolver cuánto de lo que ocurre en las cosas llamadas escuelas o que parte de las preocupaciones de una institución llamada "Ministerio de Educación" son realmente educativas y solo podemos responder manteniendo los ojos (o al menos un ojo) en lo que significa la expresión educación.

Las tentaciones (1) y (2) hacen que sea difícil para los filósofos de la educación enfrentar algunas de las preguntas más básicas que surgen espontáneamente al pensar en la educación, a saber, ¿qué es exactamente lo que la educación excluye e incluye?, ¿cuál es el uso o el valor de la educación?, ¿qué tipos de bienes produce y cómo debemos ponderar estos bienes en comparación con los bienes producidos por otras empresas?, ¿qué es aprender algo y qué tipo de cosas realmente vale la pena aprender? Estas son el tipo de preguntas que, mutatis mutandis, son abordadas por los filósofos morales, por la filosofía del derecho, de la ciencia, la estética o la política. Se trata de preguntas que son centrales con respecto a un tema en particular. No digo que los filósofos de la educación nunca se ocupen de esas cuestiones, pero es probable que las eviten porque les atrae: 1) el atractivo de algún otro tema (hoy en día generalmente político) o 2) las prácticas culturales o administrativas particulares de su propia sociedad que - aunque sean visibles o destacadas públicamente y, por lo tanto, atraen a los filósofos que quieren cortar el hielo práctico "en el mundo real" - pueden tener poco que ver con la educación y en unos pocos siglos o incluso décadas pueden no merecer más que un interés de anticuario.

\section{$\mathbf{E}$}

Mucho de lo que he dicho antes es crítico y sin duda se trata de posturas controvertidas. Si vale o no la pena decirlo depende de si uno piensa, en principio o a priori, que la filosofía de la educación tiene un papel importante que desempeñar en la teoría y la práctica de la educación. Claramente no podemos suponer menos hasta que tengamos una perspectiva adecuada sobre lo que es la filosofía de la educación como la que intento proponer. La cuestión es urgente, aunque solo sea porque la dura verdad es que la filosofía de la educación ahora importa muy poco. Rara vez se considera un componente necesario o incluso útil en la formación o educación de los educadores, de los profesores en ejercicio, de los investigadores de la educación, de los administradores o inspectores; por cierto, la idea de que los responsables de la política educativa y los políticos deban formarse en filosofía puede considerarse absurda. En este sentido, la filosofía de la educación corre el riesgo de convertirse en algo autosuficiente, escasamente institucionalizado y que solo resulta útil para aquellos que eventualmente tienen un interés particular en ella. Tal vez solo sobreviva por cortesía de quienes se resisten a abolir una actividad cuyo título, al menos, parece reivindicar cierta importancia, si no alguna utilidad o valor práctico. En comparación con su apogeo institucional (al menos en el Reino Unido) en la década de 1960 y más tarde, la filosofía de la educación tiene toda la apariencia de una especie amenazada. De modo que espero que las preguntas que he planteado aquí se consideren de forma seria, esto importa mucho más que si las respuestas que doy a ellas son adecuadas o no.

\section{Referencias}

Carr, W. (1995). For Education: towards a critical educational inquiry. Oxford University Press.

Fowler, H. W., Fowler, F. G. y Thompson, D. (1995). The concise Oxford dictionary of current English. Clarendon Press.

Gallie, W. H. (1968). Philosophy and Human Understanding. Schocken.

Hirst, P. H. y Peters, R. S. (1970). The Logic of Education. RKP.

Kohli, W. (Ed.) (1995). Critical Conversations in Philosophy of Education. RKP. 
Mills, O. (2001). ¿Who is a Philosopher of Education? In Proceedings of the Philosophy of Education Society of Great Britain. Conferencia Anual.

Partington, G. (2001). Educational contestability. Oxford Review of Education, 27(2), 205-217.

Platón. (1988). Diálogos IV. República. Gredos.
Peters, R.S. (1965). Ethics and Education. Allen y Unwin.

Peters, R.S. (ed.) (1971). The Philosophy of Education. Oxford University Press.

Wilson, J. (1979). Preface to the Philosophy of education. RKP. 